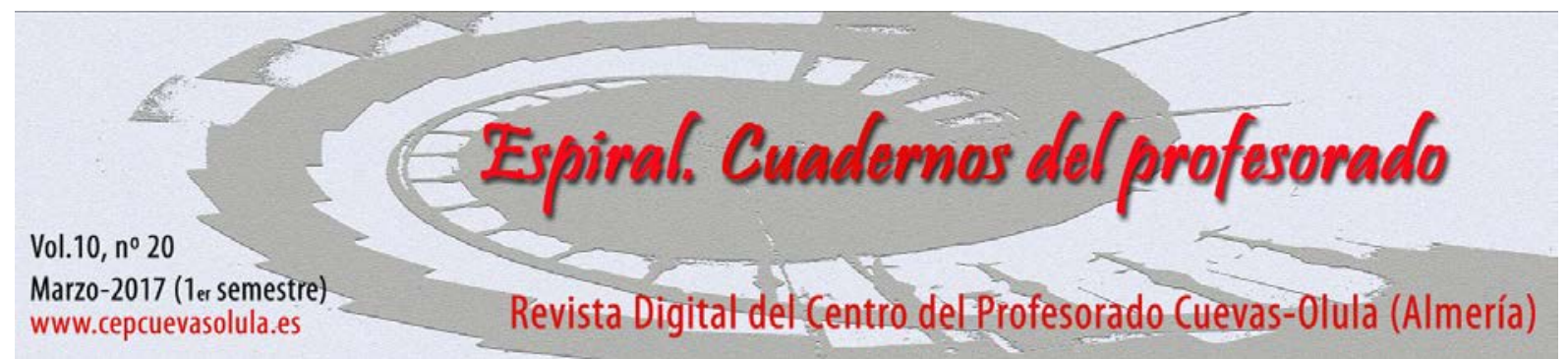

\title{
Análisis de las competencias genéricas de la Universidad de Almería en el Grado en Derecho en relación al mercado laboral
}

\section{Generic skills analysis of the University of Almeria in the Law Degree in relation to the labor market}

\author{
Esther $\mathbf{M}^{\mathrm{a}}$ Salmerón-Manzano
}

\author{
Departamento de Derecho, Universidad de Almería, CeiA3, España
}

\begin{abstract}
RESUMEN: En este estudio se analizan las 10 competencias genéricas de la Universidad de Almería, en las 37 asignaturas que componen el Grado de Derecho. Los resultados muestran respecto a las competencias que, ocupa el primer lugar la competencia de "comunicación oral y escrita en la propia lengua"; en segundo lugar, la competencia “capacidad para resolver problemas", el tercer lugar, la "capacidad de crítica y autocrítica”. Por el contrario, la competencia menos presente es la de "aprendizaje de una lengua extranjera", apareciendo solo en el $5 \%$ de las asignaturas, seguidas "competencia social y ciudadanía global”. Las competencias de habilidades en el uso de las Tecnologías de la Información y las Comunicaciones y la capacidad para aprender a trabajar de forma autónoma están por encima del 70\%, considerándose bastante adecuadas dado el gran avance que ha experimentado nuestra sociedad en los últimos años, particularmente en este tipo de habilidades. Como principal conclusión de este estudio, se recomienda que deba ser reforzada la competencia de aprendizaje de una lengua extranjera, dado que en un mundo globalizado los profesionales con conocimiento de un segundo idioma serán los más demandados, y podrán competir por empleos en el exterior.
\end{abstract}

Palabras clave: Competencias, Grado en Derecho, mercado laboral, habilidades profesionales.

ABSTRACT: In this manuscript, the 10 generic skills of UAL, in the 37 subjects comprising the Degree in Law at the University of Almeria were analysed. The results show that the most frequent skills are: first, "Oral and written communication in the own language", second, "the Ability to solve problems", third, "Capacity for critical and self-critical". On the other hand, the skills that are less present are: "Learning a foreign language", appearing in only $5 \%$ of the subjects, followed by "Social competence and global citizenship", which appears in $27 \%$ of cases. The skills of the use of Information and communication technologies and the ability to learn autonomously are above $70 \%$; that are considered quite appropriate given the great progress our society has undergone in recent years, particularly in this type of skills. As main conclusion, in this study has been detected that the skill to be strengthened is learning a foreign language; because in a globalized world professionals with knowledge of a second language will be the most popular, and can compete for jobs abroad.

Key words: Competency, Law Degree, labour market, skill.

Salmerón-Manzano, E. M. (2017). Análisis de las competencias genéricas de la Universidad de Almería en el Grado en Derecho en relación al mercado laboral. Espiral. Cuadernos del Profesorado, 10(20), 58-72. Disponible en: http://espiral.cepcuevasolula.es/

Fecha de recepción: 29/05/2016

Fecha de aceptación: 01/12/2016
Enviar correspondencia a: esthersmanza@gmail.com 


\section{Introducción}

La Universidad se debe a la sociedad en la que está inserta, respondiendo a sus demandas y necesidades (Domínguez y Baute, 2016) contribuyendo en conjunto al avance social y del conocimiento (Sobrinho, 2014). Para poder entender mejor las relaciones tanto internas como externas que se producen en el ámbito universitario se representa la figura 1 (Zabalza, 2002). Se hace así patente que la Universidad se configura en un escenario complejo y multidimensional en el que se mueven y entrecruzan influencias muy variadas (Rodríguez-Hidalgo et al., 2015). Dentro de este escenario se podrían diferenciar dos zonas: la propia Universidad constituiría un espacio interno, mientras que literalmente el resto del mundo — particularizado en la sociedad que la acogeconstituiría el espacio exterior. El espacio interior está formado por: 1. El contexto institucional, 2. Los contenidos curriculares, 3. El sector profesorado y 4 . El sector alumnado.

Por otro lado, el espacio exterior está formado por: 1. Las políticas de educación superior, 2. Los avances de la ciencia, cultura e investigación, 3. Los Colegios profesionales, y 4. El sector del empleo.

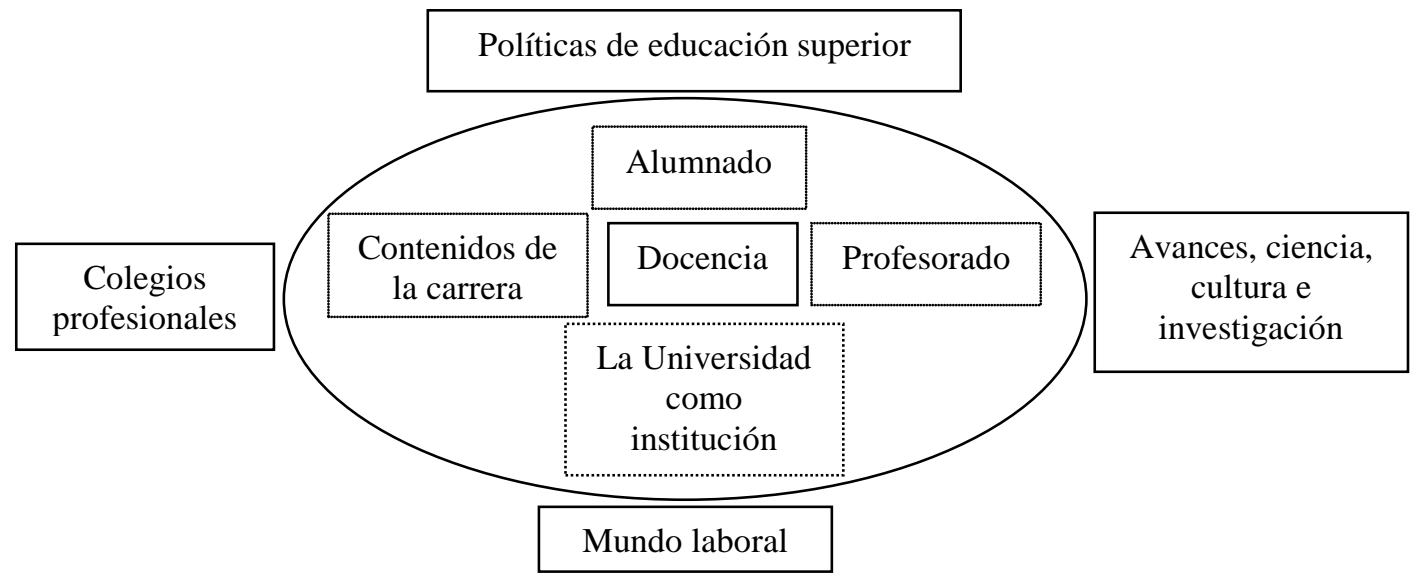

Figura 1. Modelo de interrelación de la Universidad y su entorno.

La estructura de los estudios universitarios, de acuerdo con las líneas generales emanadas del Espacio Europeo de Educación Superior y descritas en el Real Decreto 1393/2007, de 29 de octubre (BOE, 2007b), se muestran en la figura 2.

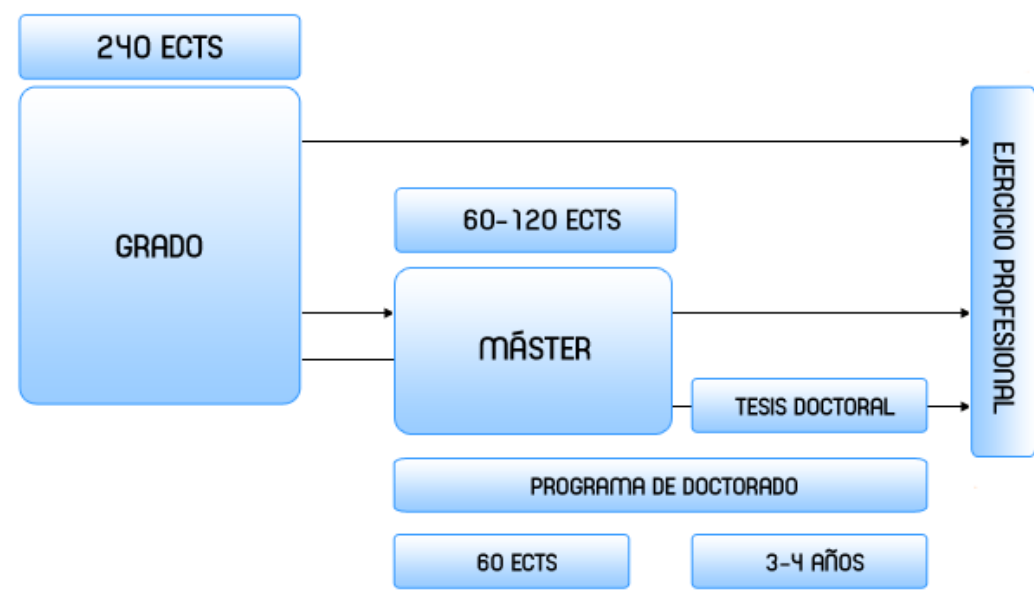

Figura 2. Estructura de las enseñanzas universitarias (BOE, 2007a) 


\section{Tabla 1}

Competencias genéricas incluidas en el Real Decreto 1393/2007, de 29 de octubre

\begin{tabular}{|c|c|c|}
\hline Código & Denominación & Competencia \\
\hline RD1 & $\begin{array}{l}\text { Poseer y } \\
\text { comprender } \\
\text { conocimientos }\end{array}$ & $\begin{array}{l}\text { Que los estudiantes hayan demostrado poseer y comprender conocimientos en un } \\
\text { área de estudio que parte de la base de la educación secundaria general, y se suele } \\
\text { encontrar a un nivel que, si bien se apoya en libros de texto avanzados, incluye } \\
\text { también algunos aspectos que implican conocimientos procedentes de la van- } \\
\text { guardia de su campo de estudio. }\end{array}$ \\
\hline RD2 & $\begin{array}{l}\text { Aplicación de } \\
\text { conocimientos }\end{array}$ & $\begin{array}{l}\text { Que los estudiantes sepan aplicar sus conocimientos a su trabajo o vocación de } \\
\text { una forma profesional y posean las competencias que suelen demostrarse por } \\
\text { medio de la elaboración y defensa de argumentos y la resolución de problemas } \\
\text { dentro de su área de estudio. }\end{array}$ \\
\hline RD3 & $\begin{array}{l}\text { Capacidad para } \\
\text { emitir juicios }\end{array}$ & $\begin{array}{l}\text { Que los estudiantes tengan la capacidad de reunir e interpretar datos relevantes } \\
\text { (normalmente dentro de su área de estudio) para emitir juicios que incluyan una } \\
\text { reflexión sobre temas relevantes de índole social, científico o ético. }\end{array}$ \\
\hline RD4 & $\begin{array}{l}\text { Capacidad de } \\
\text { comunicar y } \\
\text { aptitud social }\end{array}$ & $\begin{array}{l}\text { La elaboración y defensa de argumentos y la resolución de problemas dentro de } \\
\text { su área de estudio. }\end{array}$ \\
\hline RD5 & $\begin{array}{l}\text { Habilidad para el } \\
\text { aprendizaje }\end{array}$ & $\begin{array}{l}\text { Que los estudiantes hayan desarrollado aquellas habilidades de aprendizaje nece- } \\
\text { sarias para emprender estudios posteriores con un alto grado de autonomía. }\end{array}$ \\
\hline
\end{tabular}

Las enseñanzas universitarias oficiales están organizadas en tres ciclos (BOE, 2007c), donde el ECTS (European Credit Transfer and Accumulation System o Sistema Europeo de Transferencia y Acumulación de Créditos) es la unidad para contar la carga de trabajo que un alumno realiza: Grado (240 ECTS), Máster (60-120 ECTS) y Doctorado. Cada ciclo conduce a la obtención de un Título oficial. En todos los casos la superación del primero da acceso al segundo, y la superación del segundo da acceso al tercero. El Máster puede ser de dos tipos: (1) Máster orientado a ejercer una profesión, y (2) Máster orientado a la investigación, cuyo título permite acceder al segundo periodo de investigación del doctorado.

Las enseñanzas de Grado presentan planes de estudios de 240 créditos ECTS, y se distinguen:

1. Formación teórica y práctica: aspectos básicos de la rama de conocimiento, materias obligatorias u optativas, seminarios, prácticas externas, trabajos dirigidos, trabajos de fin de Grado (TFG), etc.

2. 60 créditos ECTS (mínimo) de formación básica, 36 vinculados a las materias de la rama concretados en asignaturas de 6 créditos o más, ofertadas en la primera mitad del plan de estudios.

3. 60 créditos ECTS (máximo) de prácticas externas.

4. Elaboración y defensa de Trabajo Fin de Grado, entre 6 y 30 créditos ECTS. Evaluación competencias asociadas a la Titulación.

5. Otras actividades universitarias, 6 créditos ECTS (máximo).

6. En el caso de títulos que habilitan para el ejercicio de actividades profesionales reguladas se debe, además de cumplir la normativa dictada por el Gobierno o, en su caso, la Disposición Transitoria $4^{\text {a }}$ del RD 1393/2007, de 29 de octubre, incluir la adquisición de las competencias necesarias para ejercer la profesión.

Los planes universitarios deben definirse para mejorar la empleabilidad (Álvarez et al. 2015); ofreciendo capacidades (Garbanzo, 2012) y competencias adecuadas para el mercado laboral (González, 2003). Además, el éxito en el mercado laboral debería ser un indicador de la calidad del rendimiento de las Universidades (Jaramillo et al., 2012), y ser recompensado en los sistemas de reglamentación, financiación y evaluación. Por tanto, se debe mostrar la pertinencia de las actividades 
universitarias, compartiendo conocimientos con la sociedad y reforzando el diálogo con todas las partes interesadas.

Tabla 2

Competencias genéricas de la Universidad de Almería

Código Denominación Competencia

Conocimientos bási- Conocimiento, habilidades y actitudes que posibilitan la comprensión de

UAL1 cos de la profesión (a nuevas teorías, interpretaciones, métodos y técnicas dentro de los diferentes completar con competencias específicas) campos disciplinares, conducentes a satisfacer, de manera óptima, las exigencias profesionales.

Utilizar las Técnicas de Información y Comunicación (TICs) como una

Habilidades en el uso
de las TIC

UAL3 solver problemas

UAL4 escrita en la propia
lengua

UAL5 Capacidad de crítica y autocrítica

UAL6 Trabajo en equipo

UAL 7 Aprendizaje de una lengua extranjera

UAL8 Compromiso ético

Capacidad para

UAL9 aprender a trabajar de forma autónoma

UAL10 Competencia social y herramienta para la expresión y la comunicación, para el acceso a fuentes de información, como medio de archivo de datos y documentos, para tareas de presentación, para el aprendizaje, la investigación y el trabajo cooperativo.

Capacidad para identificar, analizar y definir los elementos significativos que constituyen un problema, para poder resolverlo con rigor. Capacidad para aplicar los conocimientos adquiridos, así como la elaboración y defensa de argumentos y la resolución de problemas dentro de su área de estudio y/o en contextos profesionales.

Comprender, así como expresar con claridad y oportunidad las ideas, conocimientos, problemas y soluciones a un público más amplio, especializado o no especializado ( $y$ sentimientos a través de la palabra, adaptándose a las características de la situación y la audiencia para lograr su comprensión y adhesión).

Es el comportamiento mental que cuestiona las cosas y se interesa por los fundamentos en los que se asientan las ideas, acciones y juicios, tanto propios como ajenos.

Integrarse y colaborar de forma activa en la consecución de objetivos comunes con otras personas, áreas y organizaciones, en contextos tanto nacionales como Internacionales.

Entender y hacerse entender de manera verbal y escrita, usando una lengua diferente a la propia. (Especialmente importante en el proceso de Convergencia Europea, por la expansión de la dimensión internacional de las titulaciones).

Capacidad para pensar y actuar según principios de carácter universal que se basan en el valor de la persona y se dirigen a su pleno desarrollo.

Capacidad para diseñar, gestionar y ejecutar una tarea de forma personal.

Respetar los Derechos fundamentales y de igualdad entre hombres y mujeres, los Derechos Humanos, los valores de una cultura de paz y democráticos, los Principios medioambientales y de cooperación al desarrollo que promuevan un compromiso ético en una sociedad global, intercultural, libre y justa.

La universidad de este siglo busca proporcionar una educación basada en la adquisición de competencias, que aúne conocimientos, habilidades y actitudes (De Castro-Camero, 2015). Se define frecuentemente competencia como el conjunto de "conocimientos, actitudes y habilidades necesarias para desempeñar una ocupación dada” (Yániz, 2008). Sin embargo, una explicación más concreta y que compartimos plenamente es la de "sistema de conocimientos conceptuales y procedurales organizados como esquemas operacionales que permiten, frente a una familia de situaciones, la identificación de un problema y su resolución mediante una acción eficaz” (Rey, 1996). 
La Universidad de Almería ha acordado que todos los Grados deben contemplar, de forma explícita, tres tipos de competencias:

1. Las competencias genéricas incluidas en el Real Decreto 1393/2007, de 29 de octubre, por el que se establece la ordenación de las enseñanzas universitarias oficiales (Códigos RD1 a RD5) (Tabla 1).

2. Las competencias genéricas de la Universidad de Almería, aprobadas en Consejo de Gobierno de 17 de junio de 2008 (Códigos UAL1 a UAL10) (Tabla 2).

3. Las competencias específicas del título, que para el Grado en Derecho están reflejadas en el BOE de 4 de agosto de 2009.

Hay que aclarar que las competencias específicas de cada título están distribuidas entre las asignaturas que lo componen, de forma que cada universidad las tiene organizadas entre las mismas para la organización docente, y estas no se repiten, al contrario que las competencias genéricas, que la norma obliga a que estén todas presentes al menos una vez en una asignatura, pero pueden darse en tantas asignaturas como el título en su organización docente se considere oportuno. De ahí el interés de analizar planteamiento de las competencias genéricas de un título en concreto para ver qué importancia les da el título a las competencias genéricas de la propia universidad.

Explicado este contexto, y volviendo a la idea inicial de la relación de la universidad con la sociedad, lo que buscaba un egresado universitario hace unos años, era la idea de "empleo para toda la vida” y esta idea debe entenderse obsoleta, ya que hoy en día empieza a vislumbrarse el término de "empleabilidad"; donde los conocimientos son importantes, pero no lo son menos las habilidades o competencias adquiridas. Se enfatiza la responsabilidad del individuo (y de su proceso de aprendizaje) y se tienen en cuenta aspectos como el aprendizaje permanente y la movilidad. Por ejemplo, en el curso 2015-16, se admitieron 90 solicitudes de alumnos del grado de derecho en la Universidad de Almería para realizar una estancia Erasmus con destinos tan dispares como: Italia, Alemania, Francia, Polonia, Eslovaquia o Lituania, para cubrir un total de 91 plazas ofertadas, donde la mayoría son para un curso completo 9 o 10 meses. Destaca Italia como destino por su oferta, dada la similitud del derecho italiano con el español. No obstante, en los exámenes de idioma de los estudiantes de derecho para la convocatoria erasmus, muestran masivamente su preferencia por realizar la prueba de idioma en inglés.

Los estudios de derecho tienen una demanda aceptable en nuestro país, tal como lo demuestran las últimas estadísticas publicadas por el Ministerios de Educación (MECD, 2016) para el curso 2014-15, donde la admisión en el grado de derecho en la universidad española tiene una nota de corte de 8,6 si se procede de PAU (Prueba de Acceso a la Universidad) y de 7,48 si por FP (Formación Profesional). Estando por encima de titulaciones de la rama de economía (8,52 PAU, 7,45 FP), arquitectura y construcción (8,31 PAU, 6,99 FP) e incluso informática (7,90 PAU, 7,22 FP). Sin embargo, es una titulación con una tasa de rendimiento (en el Grado) según el número de créditos aprobados del $70 \%$, más elevada que por ejemplo Economía 67,8\% o Informática $61,8 \%$. Con estas cifras cabría pensar una buena perspectiva para los egresados, sin embrago no ocurre así. Si analizamos la tasa de afiliación de los egresados universitarios en derecho cuatro años después de finalizar los estudios, con datos de 2014 y cohorte de egresados en el curso 2009-2010, observamos que es del 49,4\%, la más baja de todo el sistema universitario español, siendo por ejemplo la de informática del 78,0\% la más elevada.

Respecto a la empleabilidad (MECD, 2016), hay que distinguir como principales opciones en régimen de autónomos y por cuenta ajena. En régimen de autónomos los titulados en derecho tienen un 15,6\%, mientras que otras como informática tienen el 6,5 \%, siendo los mayores niveles para los titulados en arquitectura y construcción (27,5\%) y en veterinaria con el $26.4 \%$. Respecto a la contratación por cuenta ajena en derecho están en el $70 \%$, mientras que el máximo esta en informática con el $72 \%$. Por último, queda analizar si estos egresados universitarios realizan un trabajo acorde a su nivel formativo cuatro años después de finalizar los estudios, aquí los titulados en derecho están al $57,5 \%$, mientras que por ejemplo Informática es del 49,9\%. Siendo el mayor de ellos los titulados en salud 87,3\% y el menor los titulados Administración y Negocios con el 31,8\%. 
Tabla 3

Asignaturas por módulos del Grado en Derecho

\begin{tabular}{|c|c|c|c|c|}
\hline Área/módulo & $\mathbf{N}^{\mathbf{0}}$ & Asignatura & ECTS & Carácter \\
\hline \multirow{3}{*}{ Ciencias Jurídicas Básicas } & 1 & Derecho Romano & 8 & básica \\
\hline & 2 & Historia del Derecho & 8 & básica \\
\hline & 3 & Teoría del Derecho & 8 & básica \\
\hline \multirow{4}{*}{ Derecho Civil } & 4 & Derecho de Familia y Sucesiones & 6 & obligatoria \\
\hline & 5 & Derecho de Obligaciones y Contratos & 8 & obligatoria \\
\hline & 6 & Derechos Reales y Registro de la Propiedad & 7 & obligatoria \\
\hline & 7 & Introducción al Derecho Civil & 6 & básica \\
\hline Economía y Empresa & 8 & Introducción a la Economía & 6 & básica \\
\hline \multirow{4}{*}{$\begin{array}{l}\text { Derecho Constitucional, de } \\
\text { la Unión Europea y } \\
\text { Libertades }\end{array}$} & 9 & Derecho Constitucional Avanzado & 9 & obligatoria \\
\hline & 10 & Derecho Constitucional Básico & 9 & básica \\
\hline & 11 & Derecho Eclesiástico del Estado & 4 & obligatoria \\
\hline & 12 & Instituciones y Derecho de la Unión Europea & 8 & obligatoria \\
\hline \multirow{2}{*}{ Derecho Administrativo } & 13 & Derecho Administrativo Especial & 9 & obligatoria \\
\hline & 14 & Derecho Administrativo General & 9 & básica \\
\hline \multirow{3}{*}{ Derecho Penal } & 15 & Derecho Penal Español: Parte Especial I & 6 & obligatoria \\
\hline & 16 & Derecho Penal Español: Parte Especial II & 6 & obligatoria \\
\hline & 17 & Derecho Penal Español: Parte General & 6 & básica \\
\hline \multirow{3}{*}{ Derecho Procesal } & 18 & Derecho Procesal Civil & 6 & obligatoria \\
\hline & 19 & Derecho Procesal Penal & 6 & obligatoria \\
\hline & 20 & Introducción al Derecho Procesal & 6 & básica \\
\hline \multirow{4}{*}{ Derecho de la Empresa } & 21 & Contratación Mercantil y Derecho Concursal & 9 & obligatoria \\
\hline & 22 & $\begin{array}{l}\text { Derecho colectivo y Derecho público del } \\
\text { Trabajo }\end{array}$ & 6 & obligatoria \\
\hline & 23 & $\begin{array}{l}\text { Derecho del Empresario y las Sociedades } \\
\text { Mercantiles }\end{array}$ & 9 & obligatoria \\
\hline & 24 & Derecho del Trabajo y de la Seguridad Social & 6 & obligatoria \\
\hline \multirow{3}{*}{ Derecho Internacional } & 25 & Derecho del Comercio Internacional & 4 & obligatoria \\
\hline & 26 & Derecho Internacional Privado & 8 & obligatoria \\
\hline & 27 & Derecho Internacional Público & 9 & obligatoria \\
\hline \multirow[t]{2}{*}{ Derecho Financiero } & 28 & Derecho Financiero & 9 & obligatoria \\
\hline & 29 & Derecho Tributario Especial & 9 & obligatoria \\
\hline \multirow{5}{*}{ Prácticas y Fin de Grado } & 30 & Itinerario de Práctica Jurídica Privada & 6 & obligatoria \\
\hline & 31 & Itinerario de Práctica Jurídica Pública & 6 & obligatoria \\
\hline & 32 & $\begin{array}{l}\text { Itinerario de Prácticas en Administración y } \\
\text { Función pública }\end{array}$ & 6 & obligatoria \\
\hline & 33 & Itinerario de Prácticas en Empresas & 6 & obligatoria \\
\hline & 34 & Prácticas Externas & 12 & $\begin{array}{l}\text { prácticas } \\
\text { externas }\end{array}$ \\
\hline \multirow[t]{2}{*}{ Optatividad } & 35 & Democracia, Ciudadanía y Globalización & 6 & optativa \\
\hline & 36 & Derechos Humanos & 6 & optativa \\
\hline
\end{tabular}




\section{La Titulación de Derecho en la Universidad de Almería}

La titulación de Derecho es una de las de mayor tradición y, en la actualidad, se imparte en todas las Universidades españolas. Estudiar Derecho significa conocer y aprender a aplicar las normas jurídicas con el razonamiento que es propio de un jurista. Aprender los conocimientos jurídicos, la interpretación de las normas, el análisis crítico de los problemas y la capacidad para exponer y razonar diversas soluciones a las mismas cuestiones son, entre otras, las distintas competencias y habilidades que ha de adquirir el alumno en nuestras aulas. De ahí que su formación requiera, de forma inicial, superar los contenidos de las distintas asignaturas, aunque éste no sea sólo el núcleo principal de su formación. Y es que, a la formación científica adecuada en los aspectos básicos del Derecho vigente, se ha de unir la formación sobre las técnicas necesarias para su aplicación práctica. El antecedente al actual Grado en Derecho fue el Título de Licenciado en Derecho, plan de 1953 (aprobado por Decreto de 11 de agosto, BOE 29.08.1953). Su estructura y contenido satisface las necesidades que exige la formación de un jurista. En el caso de los Planes de estudio de Derecho, los cambios basados en la implantación del EEES y las competencias están permitiendo el diseño del proceso curricular de las diferentes profesiones jurídicas en Europa, permitiendo una plena intercambiabilidad en la formación y una optimización del ejercicio profesional, cualquiera que sea el Estado de origen en el cual se hayan cursado los estudios en Derecho (León, 2007).

La licenciatura en derecho en España, hasta la llegada del grado, no había sufrido reformas sustanciales desde el plan de estudios de 1957, por ejemplo, en la Universidad de Almería estuvo en vigor hasta la entrada en vigor del grado (plan de estudio 2010). En la tabla 4, se muestra una tabla de equivalencias, que se emplea para convalidar aquellas las materias cursadas en la licenciatura en derecho, en relación al actual grado de derecho. Y que se emplea para la adaptación de aquellos alumnos que no finalizaron sus estudios en el plan de 1957. No obstante, hay que aclarar que en ningún caso las licenciaturas equivalen al nivel de grado, por normal general lo hacen al de máster. En concreto para la licenciatura en derecho está regulado por Resolución de 14 de septiembre de 2015, de la Dirección General de Política Universitaria, por la que se publica el Acuerdo del Consejo de Ministros de 4 de septiembre de 2015, por el que se determina el nivel de correspondencia al nivel del Marco Español de Cualificaciones para la Educación Superior del Título Universitario Oficial de Licenciado en Derecho. En él se especifica que El Real Decreto 1027/2011, de 15 de julio, por el que se establece el Marco Español de Cualificaciones para la Educación Superior, establece cuatro niveles de cualificación en función de los resultados de aprendizaje que proporcionan los estudios oficiales: el nivel de Técnico Superior se incluye en el Nivel 1, el de Grado en el nivel 2, el de Máster en el nivel 3, y el de Doctor en el nivel 4. Y más adelante se especifica que "El 30 de abril de 2015, la Agencia Nacional de Evaluación de la Calidad y de la Acreditación evacuó el informe para la determinación de la correspondencia del título de Licenciado en Derecho. En este informe se indica que la titulación universitaria de Licenciado en Derecho se corresponde con el nivel 3 del MECES (nivel de máster)”. Esto implica, por ejemplo, que los licenciados en derecho puedan acceder al doctorado directamente sin necesidad de realizar ningún Máster, cosa que si debe realizar los graduados en derecho.

El Título de Grado en Derecho por la Universidad de Almería, se aprobó en el BOE de 1 de noviembre de 2011 (BOE, 2011) y se implantó en el curso 2010-11 no sufriendo ninguna modificación posterior en el BOE. Consta de una oferta total de 264 ECTS, de los cuales el estudiante deberá cursar 240 ECTS. Cada crédito ECTS se corresponde con un valor de 25 horas de trabajo del estudiante, por lo que un curso completo requiere una dedicación de 1500 horas (25 horas x 60 créditos ECTS). La programación de las materias y su coordinación por cursos pretende garantizar que estas horas se distribuyan uniformemente a lo largo de las 40 semanas/anuales del período lectivo del calendario académico. La estructura completa se muestra en la tabla 3. El título distingue entre el carácter de las asignaturas básicas y obligatorias, esto debe entenderse que las básicas responden como en todos los títulos a un mínimo de 60 créditos comunes entre disciplinas de la rama del conocimiento, por ejemplo, en las carreras de ingeniería, la formación básica responde a las materias básicas que deben cursar todas las carreras de ingeniería independientemente de su posterior orientación agrícola, industrial o aeronáutica, por ejemplo. Hay que aclarar que los títulos a veces hacen referencia a las competencias 
básicas, pero en este caso se refieren a las competencias del Real Decreto 1393/2007, esto se debe a que el impreso normalizado de la ANECA para la verificación de los títulos se refiere a competencias básicas y generales para referirse a las del Real Decreto mencionado.

El estudiante en $4^{\circ}$ curso de derecho en la Universidad de Almería deberá cursar una de las cuatro asignaturas obligatorias del módulo de formación jurídica complementario, orientada a la práctica:

- Itinerario de práctica jurídica privada,

- Itinerario de práctica jurídica pública,

- Itinerario de prácticas en Administración y Función pública, o

- Itinerario de prácticas en empresas.

Teniendo en cuenta que:

- Son excluyentes. Por tanto, sólo se puede cursar una de ellas.

- Será requisito para poder superar la asignatura elegida, que el estudiante haya superado 162 créditos.

Aunque la norma permite que los títulos de Grado puedan incorporar menciones alusivas a itinerarios o intensificaciones curriculares de modo que el estudiante pueda acabar alcanzando una determinada especialización; el título de Grado en Derecho por la Universidad de Almería, no contempla en su plan de estudios ninguna mención. Hay que aclarar que todos los títulos de grado deben recoger un módulo de prácticas en empresa, pero para solventar la situación de que no existan suficientes empresas para realizar el periodo de prácticas, existen asignaturas suplementarias a las mismas; a estas en el grado de derecho se les han denominado itinerarios, pero no suponen ninguna mención en el título.

El estudiante en $4^{\circ}$ curso debe cursar 6 créditos de optatividad, pudiendo elegir entre las dos asignaturas optativas ofertadas: Democracia, Ciudadanía y Globalización, o Derechos humanos.

\section{Tabla 4}

Equivalencias establecidas en la Universidad de Almería entre la licenciatura en Derecho y el Grado de Derecho

\begin{tabular}{ll}
\hline Grado en Derecho (Plan 2010) & Licenciado en Derecho (Plan 1953) \\
\hline Derecho Romano & Derecho Romano \\
Historia del Derecho & Historia del Derecho Español \\
Teoría del Derecho & Derecho Natural \\
Teoría del Derecho & Filosofía del Derecho \\
Derecho Constitucional Básico & Derecho Político I \\
Derecho Constitucional Avanzado & Derecho Político II \\
Introducción a la Economía & Economía Política \\
Derecho Eclesiástico del Estado & Derecho Eclesiástico del Estado \\
Introducción al Derecho Civil & Derecho Civil I \\
Derecho Penal Español: Parte General & Derecho Penal I \\
Derecho Penal Español: Parte Especial I & Derecho Penal II \\
Derecho Penal Español: Parte Especial II & \\
Derecho de Obligaciones y Contratos & Derecho Civil II
\end{tabular}




\section{Tabla 4}

Equivalencias establecidas en la Universidad de Almería entre la licenciatura en Derecho y el Grado de Derecho

\begin{tabular}{ll}
\hline Grado en Derecho (Plan 2010) & Licenciado en Derecho (Plan 1953) \\
\hline Derecho Internacional Público & Derecho Internacional Público \\
Derecho Administrativo General & Derecho Administrativo I \\
Derecho Financiero & Derecho Financiero I \\
Derecho Administrativo Especial & Derecho Administrativo II \\
Derecho Tributario Especial & Derecho Financiero II \\
$\begin{array}{l}\text { Derecho Colectivo y Derecho Público del Trabajo } \\
\text { Derecho del Trabajo y de la Seguridad Social }\end{array}$ & Derecho del Trabajo \\
Derecho del Empresario y las Sociedades Mercantiles & \\
Derechos Reales y Registro de la Propiedad & Derecho Mercantil I \\
Introducción al Derecho Procesal & Derecho Civil III \\
Contratación Mercantil y Derecho Concursal & Derecho Procesal I \\
Derecho Procesal Penal & Derecho Mercantil II \\
Derecho de Familia y Sucesiones & Derecho Procesal II \\
Derecho Procesal Civil & Derecho Civil IV \\
Derecho Procesal Penal & Derecho Procesal I \\
Introducción al Derecho Procesal & Derecho Procesal II \\
Derecho Internacional Privado & \\
\hline
\end{tabular}

\section{Objetivos}

El libro blanco de la ANECA en relación al título de grado en derecho (ANECA, 2005), recoge para los siguientes perfiles profesionales: Despachos de abogados, Procuradores, Notarios, Registradores, Juzgados de $1^{\text {a }}$ Instancia, Ministerio Fiscal, Secretarios de Juzgados, Funcionarios de Administración Pública, Escala Superior Funcionarios de Administración Local, Funcionarios de Escala Media Unión Europea, Banca, Gran Empresa, Asesorías, Consultorías, Sindicatos, ONG (organización no gubernamental), y Otras asociaciones; tres tipos de competencias, las cuales son valoradas por este orden según las profesiones descritas: $1^{\circ}$ conocimientos disciplinares (saber), $2^{\circ}$ competencias profesionales (saber hacer) y $3^{\circ}$ competencias académicas.

Por ello debemos entender las competencias profesionales como el conjunto de técnicas, habilidades y destrezas específicas aplicadas al ejercicio de una profesión concreta y distinguirlas de Conocimientos Disciplinares (hacer) que vienen determinadas por el conjunto de conocimientos prácticos requeridos para involucrarse en cada uno de los sectores profesionales singularizados dentro del ámbito jurídico (en este trabajo son las competencias específicas del título de grado en derecho) o de las Competencias Académicas que están constituidas por el bagaje de conocimientos teóricos aprehendidos por el licenciado a lo largo de su formación universitaria (en este trabajo son las competencias del Real Decreto).

Por tanto, el objetivo de este estudio es analizar la distribución de las competencias genéricas o transversales de la Universidad de Almería en título de Grado en Derecho, de su distribución por materias y áreas de conocimiento asignadas a dichas asignaturas de cara a que un estudiante de Grado en Derecho adquiera las habilidades necesarias para satisfacer el mercado laboral actual, es decir las competencias profesionales (saber hacer). 


\section{Metodología}

Para poder alcanzar el objetivo propuesto se han estudiado todas las guías docentes del Grado en Derecho en relación con las 10 competencias genéricas de la Universidad de Almería. Estas son las siguientes a las cuales se las ha codificado como en el plan de estudios para facilitar la comprensión de las tablas y figuras: 1 Conocimientos básicos de la profesión (UAL1), 2 Habilidades en el uso de las TIC (UAL2), 3 Capacidad para resolver problemas (UAL3), 4 Comunicación oral y escrita en la propia lengua (UAL4), 5 Capacidad de crítica y autocrítica (UAL5), 6 Trabajo en equipo (UAL6), 7 Aprendizaje de una lengua extranjera (UAL7), 8 Compromiso ético (UAL8), 9 Capacidad para aprender a trabajar de forma autónoma (UAL9), 10 Competencia social y ciudadanía global (UAL10).

Para ello se ha realizado una consulta web al plan de estudios del grado de derecho de la Universidad de Almería (http://cms.ual.es/UAL/estudios/grados/plandeestudios/GRADO0710), donde se ha consultado para cada asignatura la guía docente del curso 2016-2017, y de ellas se ha extraído en una hoja Excel que competencias genéricas tienen recogidas. En la figura 3 se presenta un diagrama de flujos con la metodología empleada.

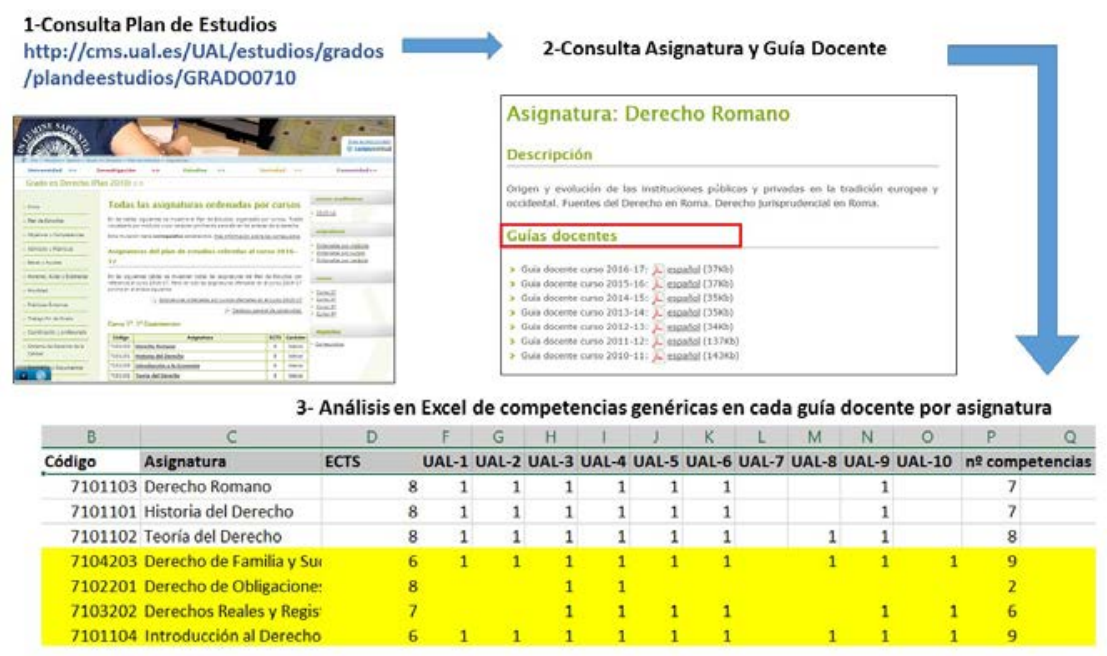

Figura 3. Metodología empleada en el estudio.

\section{Resultados}

En la figura 3 se exponen los resultados de la evaluación del número de competencias por asignatura, referidas a la tabla 3 . Se observa que sólo hay dos asignaturas que tienen incluidas las 10 competencias, siendo éstas Derecho Internacional Público y Derecho Tributario Especial. La media por asignatura es de 6,8, es decir, que cada disciplina contempla entre 6 y 7 competencias genéricas, lo cual se considera bastante elevado. La excepción está en dos asignaturas que tienen en su guía docente solo dos competencias. Estás son Derecho de Obligaciones y Contratos junto a Derecho Constitucional Básico. En relación a esta última, ya hay estudios específicos que piden aumentar el número de competencias de esta materia en España (Saldaña, 2011).

Atendiendo a las competencias en concreto, podemos observar, figura 4 referida a la tabla 2, que ninguna competencia está en el $100 \%$ de las asignaturas. No obstante, hay algunas competencias que, en la titulación de Derecho, se contemplan como muy prioritarias dada la frecuencia con la que se reflejan en las distintas guías docentes. Estas son, en primer lugar, la UAL-4 Comunicación oral y escrita en la propia lengua, con una frecuencia del 97,2 \%, es decir que aparece en 35 de las 36 asignaturas posibles. Este resultado es muy esperable para esta titulación, dada las perspectivas profesionales que presenta, por ejemplo el ejercicio libre de la profesión como Abogado, donde la comunicación escrita es la herramienta básica para el desempeño de esta labor. Sin embargo, para estos profesionales, la comunicación oral resulta también imprescindible, valiendo de ejemplo para la 
celebración de vistas orales de juicios, así como algo tan necesario como la entrevista con un cliente potencial. En segundo lugar, destaca la competencia UAL-3, Capacidad para resolver problemas, la cual resulta también bastante usual en todas las titulaciones universitarias. En tercer lugar, está la competencia UAL-5, Capacidad de crítica y autocrítica, que también es muy común en todas las titulaciones españolas, incluidas las de ciencias (de Castro-Camero, 2015). Por el contrario, existen competencias poco habituales en el Grado en Derecho. Así, encontramos en último lugar la UAL-7, Aprendizaje de una lengua extranjera, que sólo aparece en 2 asignaturas en toda la titulación. Sin tratar de justificar esta cuestión, pero entendiendo que la legislación española difiere bastante de la sajona o la germánica, es de entender que textos escritos en otros idiomas son de un interés más relativo para un jurista en España, por lo que no resulta sorprendente este resultado. Sin embargo, desde el punto de vista laboral y el investigador, es una limitación clara para competir por empleos en el exterior o el trabajo en empresas multinacionales, donde el conocimiento de una segunda lengua puede resultar un hándicap. Lo que si llama la atención en este estudio es que ocupe el penúltimo lugar la competencia UAL-10, Competencia social y ciudadanía global, puesto que se ocupa del estudio del respeto a los derechos fundamentales y de igualdad entre hombres y mujeres; los Derechos Humanos; los valores de una cultura de paz y democráticos; los principios medioambientales y de cooperación al desarrollo que promuevan un compromiso ético en una sociedad global, intercultural, libre y justa. Todo ello parece muy inherente a la propia titulación de Derecho. No obstante, esta posición la atribuimos a que las competencias deben ser evaluadas, y en la guía docente debe tener un peso en la calificación global al igual que las competencias propias de la titulación asignadas a la asignatura, siendo esta competencia centrada en los valores, generalmente, difícil de evaluar de forma objetiva.

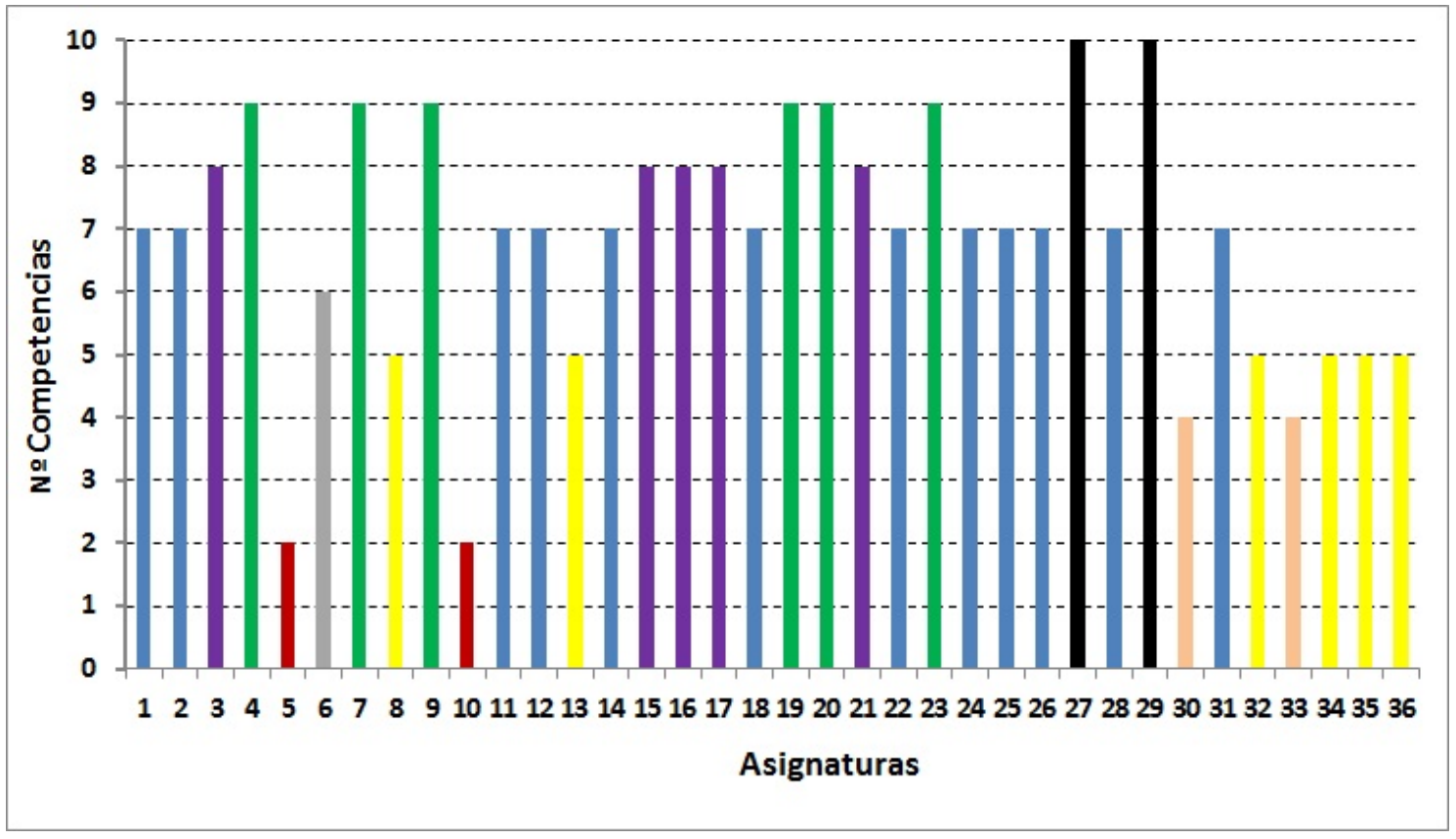

Figura 4. Número de competencias por asignaturas.

En la figura 5 se muestran los resultados de las competencias en función del número de créditos totales por los que se ven afectados; donde se observa la misma tendencia que por número de asignaturas. Ello se debe a que la variabilidad de los créditos por asignatura no influye en este resultado. Si el análisis se centra en áreas de conocimiento o módulos según el plan de estudios, obtenemos la tabla 4, donde se han agrupado por áreas las competencias y se han promediado, ofreciendo los resultados en tanto por ciento. En general, se observa que las asignaturas que pertenecen a un área de conocimiento son bastante regulares en la asignación de competencias genéricas a sus materias, exceptuando la optatividad, lo cual es obvio dada la naturaleza de las asignaturas. 


\section{Discusión}

Aclarado que la licenciatura en Derecho equivale al nivel de Máster (BOE, 2015). En relación al cambio que supone pasar de la licenciatura en derecho al grado, para el ejercicio de la profesión, hay que recoger el análisis realizado para esto realizado para la Universidad Autónoma de Madrid en relación al grado de derecho (DíezHochleitner y Rodríguez, 2007): "Lo que tradicionalmente se ha aprendido en la carrera de Derecho, evidentemente, se seguirá aprendiendo en el nuevo Grado. No obstante, el nuevo plan descarta la pretensión (tan enraizada hasta ahora entre los profesores) de querer transmitir de forma exhaustiva el conocimiento jurídico y se preocupa por asegurar una sólida formación básica del alumno (que más tarde se completará en el marco de un posgrado especializado o del ejercicio profesional). Por otra parte, el plan de estudios incluye actividades y materias destinadas a la adquisición de las habilidades y destrezas que requiere un profesional del Derecho". Y la conclusión final de este trabajo que es aplicable a todos los grados en general y a los de derecho en particular, el objetivo de los grados es "acercar el diseño de los planes de estudio a las demandas de nuestro entorno social -y, por supuesto, profesional-, exigir del estudiante que trabaje 40 horas a la semana (también puede cursar los estudios a tiempo parcial, durante más años) o prever prácticas externas obligatorias”. En resumen, los títulos de grados buscan fomentar las competencias más que los conocimientos. Por ello es tan importante evaluar las competencias que pretenden desarrollar y fomentar los planes de estudio de los grados.

En este trabajo se han analizado las competencias genéricas de la Universidad de Almería en relación al Grado en Derecho. Los resultados obtenidos muestran que, en general, las distintas áreas de conocimiento suelen tener las mismas competencias en sus guías docentes, a excepción de la optatividad. En relación a las competencias por asignaturas, se ha visto como destaca la competencia de Comunicación oral y escrita en la propia lengua, con una frecuencia del 97,2 \%, y queda claro que así debe ser por las salidas profesionales más naturales de esta titulación. En segundo lugar, al igual que muchas de las titulaciones de Grado españolas, está la Capacidad para resolver problemas, que es necesario que se demande a un titulado universitario. Por otro lado, se ha visto que la competencia de Aprendizaje de una lengua extranjera está muy poco presente, habiendo sido observada en sólo 2 asignaturas en toda la titulación, y aunque ello pueda estar justificado, dada la relativa relevancia de textos escritos en otros idiomas para los juristas españoles, puesto que en un mundo globalizado los profesionales con conocimiento de un segundo idioma serán los más demandados y podrán competir por empleos en el exterior, sería deseable reforzar la competencia de aprendizaje de una lengua extranjera en el título de Grado en Derecho de la Universidad de Almería, dada la gran vocación de comercio exterior de la provincia y la numerosa afluencia de ciudadanos extranjeros que acuden a la misma, bien por turismo bien por trabajo. Por otro lado, aunque el plan de estudios no recoja en demasía la competencia del segundo idioma, si parece que los estudiantes del grado en derecho complementan esta aparente carencia con la realización de estancias en el extranjero, tal como lo demuestran las cifras de estudiantes de derecho que han realizan estancia erasmus en el curso 2015-16.

El análisis de las competencias genéricas de las universidades se ha realizado en algunos trabajos, por ejemplo, comparando las competencias genéricas entre universidades andaluzas del Ceia3 (Manzano-Agugliaro et al., 2016), donde se incluye el título de grado de derecho en todas ellas y donde se observa gran discrepancia en el número de competenciales genéricas entre universidades, oscilando por ejemplo entre las tres de la Universidad de Córdoba y las diecisiete de la Universidad de Espiral. Cuadernos del Profesorado. ISSN 1988-7701 2017, vol. 10 , n ${ }^{\circ} 20$, pp. $58-72$ 
Huelva; y donde la universidad de Almería tiene un numero intermedio, diez competencias transversales. En este trabajo se podía apreciar que en el bloque de las competencias instrumentales estas eran las menos seleccionadas por las universidades, donde la más frecuente, sólo lo está en dos universidades como máximo, "la capacidad de análisis y síntesis” o las de organizar y planificar”; esto sin embargo en el grado de derecho si está en gran número de asignaturas, en el 91,7 \% (la competencia UAL5). Por otro lado las competencias relativas a los valores, tal es el caso de la competencia del "Compromiso ético" está presente sólo en 3 de las 5 universidades del CEIA3 (Campus de Excelencia Agroalimentario 3); aunque esta última está recogida en el grado de derecho por estarlo en las competencias genéricas de la Universidad de Almería, es la tercera competencia menos frecuente, apareciendo sólo en el $41,7 \%$ de las asignaturas.

Por otro lado, el análisis de las competencias genéricas de la Universidad de Almería sus titulaciones, se ha realizado para los grados de Grado en Ciencias Ambientales (Plan 2009) (Giménez et al., 2016) y de Grado en Química (Plan 2009) (Garrido-Cárdenas et al., 2016). En el primero se observó que sólo una asignatura del grado tenía las 10 competencias transversales, que sólo 3 tenían más de 5 , y que las competencias más frecuentes con porcentaje superior al $65 \%$ eran las UAL4 (Comunicación oral y escrita en la propia lengua) y la UAL6 (trabajo en equipo). En el segundo título analizado se observó que, igual que en el grado anterior sólo había una asignatura con todas las competencias transversales; las competencias más frecuentes eran las UAL3 (Capacidad para resolver problemas) en el $50 \%$ de las asignaturas y la UAL6 (Trabajo en equipo) en el $47.2 \%$ de los casos. Ambos trabajos concluyen que no se pone especial interés en la competencia del segundo idioma (UAL7), y coinciden en la importancia que se le da al trabajo en equipo (UAL6).

\section{Tabla 5}

Competencias por áreas de conocimiento (\%)

\begin{tabular}{lccccccccccc}
\hline \multirow{1}{*}{ Área/modulo } & UAL- & UAL- & UAL- & UAL- & UAL- & UAL- & UAL- & UAL- & UAL- & UAL- \\
& $\mathbf{1}$ & $\mathbf{2}$ & $\mathbf{3}$ & $\mathbf{4}$ & $\mathbf{5}$ & $\mathbf{6}$ & $\mathbf{7}$ & $\mathbf{8}$ & $\mathbf{9}$ & $\mathbf{1 0}$ \\
\hline Ciencias Jurídicas Básicas & 100 & 100 & 100 & 100 & 100 & 100 & 0 & 33 & 100 & 0 \\
Derecho Civil & 50 & 50 & 100 & 100 & 75 & 75 & 0 & 50 & 75 & 75 \\
Economía y Empresa & 100 & 0 & 100 & 100 & 100 & 0 & 0 & 0 & 100 & 0 \\
Derecho Constitucional, de la & 75 & 75 & 75 & 75 & 100 & 75 & 0 & 25 & 100 & 25 \\
Unión Europea y Libertades & & & & & & & & & \\
Derecho Administrativo & 100 & 50 & 100 & 100 & 50 & 50 & 0 & 50 & 100 & 0 \\
Derecho Penal & 100 & 100 & 100 & 100 & 100 & 100 & 0 & 100 & 100 & 0 \\
Derecho Procesal & 100 & 100 & 100 & 100 & 100 & 100 & 0 & 67 & 100 & 67 \\
Derecho de la Empresa & 100 & 100 & 100 & 100 & 100 & 100 & 0 & 50 & 100 & 25 \\
Derecho Internacional & 100 & 100 & 100 & 100 & 100 & 100 & 33 & 33 & 100 & 33 \\
Derecho Financiero & 100 & 100 & 100 & 100 & 100 & 100 & 50 & 100 & 100 & 50 \\
Prácticas y Fin de Grado & 100 & 20 & 100 & 100 & 100 & 20 & 0 & 0 & 60 & 0 \\
Optatividad & 50 & 100 & 50 & 100 & 50 & 50 & 0 & 50 & 50 & 50 \\
\hline
\end{tabular}

\section{Conclusiones}

Estudiado el mercado laboral en España en relación a los graduados en derecho, y teniendo en cuenta que sólo la mitad de estos egresados universitarios realizan un trabajo acorde a su nivel formativo cuatro años después de finalizar los estudios. Se obtiene como primera conclusión, que debería proponer en los títulos universitarios la competencia de liderazgo para revertir esta situación. Además, es necesario reflexionar y hacer hincapié en mejorar la evaluación de las competencias 
transversales, entendidas como competencias profesionales (saber hacer), que por otro lado son las demandadas por los empleadores. Habría que hacer un esfuerzo en el sistema universitario por unificar criterios en la evaluación de dichas competencias y establecer un sistema de indicadores en los títulos de grado en general y en el de derecho en particular, para poder establecer instrumentos que permitan conocer e inferir un seguimiento de la repercusión de los mismos en términos de empleabilidad. Con este nuevo enfoque se invita a reflexionar a los responsable universitarios sobre las competencias profesionales demandadas por los empleadores, para que las universidades, establezcan las estrategias pertinentes para adaptarse a las necesidades futuras de la sociedad mediante la redefinición, evaluación y seguimiento de las competencias profesionales, y de este modo cumplir con las grandes expectativas que actualmente tiene el título de grado en derecho en el sistema universitario español

\section{Referencias}

ANECA. 2005. Libro blanco del Título Grado en derecho. Disponible en: http://www.aneca.es/var/media/150240/libroblanco_derecho_def.pdf

Álvarez Pérez., A., González Afonso, M., y López Aguilar D. (2015). La Enseñanza Universitaria Y La Formación Para El Trabajo. Un análisis desde la opinión de los estudiantes. Paradigma, 30(2), 7-19.

BOE. 2007a. Orden ECI/2514/2007, de 13 de agosto, sobre expedición de títulos universitarios oficiales de Máster y Doctor. Boletín Oficial del Estado no 200. Publicado 21/08/2007, 35424-35431.

BOE. 2007b. Real decreto 1393/2007, de 29 de octubre, por el que se establece ordenación de las enseñanzas universitarias oficiales. Boletín Oficial del Estado nº 260. Publicado 30/10/2007, 44037-44048.

BOE. 2007c. Real Decreto 900/2007, de 6 de julio, por el que se crea el Comité para la definición del Marco Español de Cualificaciones para la Educación Superior. Boletín Oficial del Estado nº 172. Publicado 19/07/2007, 31371-31372.

BOE. 2011. Resolución de 7 de octubre de 2011, de la Universidad de Almería, por la que se publica el plan de estudios de Graduado en Derecho.

BOE. 2015. № 236 de viernes 2 de octubre de 2015. Resolución de 14 de septiembre de 2015, de la Dirección General de Política Universitaria, por la que se publica el Acuerdo del Consejo de Ministros de 4 de septiembre de 2015, por el que se determina el nivel de correspondencia al nivel del Marco Español de Cualificaciones para la Educación Superior del Título Universitario Oficial de Licenciado en Derecho.

De Castro-Camero, R. (2015). Los Estudios Universitarios en España y el Aprendizaje basado en la Adquisición de Competencias, Educación y Territorio, 4(1), 82-93.

Díez-Hochleitner, J., y Rodríguez de Santiago J. M. (2007). El proceso de Bolonia y el nuevo plan de estudios de Derecho de la UAM. Actualidad Jurídica, 18, 131-147.

Domínguez Domínguez, M., y Baute Álvarez, L. M. (2016). Algunas regularidades del componente laboral en las carreras universitarias. Revista Universidad y Sociedad, 8(1), 45-49.

Garbanzo Vargas, G. (2012). Factores asociados al rendimiento académico en estudiantes universitarios, una reflexión desde la calidad de la educación superior pública. Revista Educación, 31(1), 43-63.

Garrido-Cárdenas J. A., Giménez E., Salmerón-Manzano E., y Manzano-Agugliaro F. (2016). Generic skills assessment in the chemistry degree at University of Almeria. ICERI 2016. 9th annual International Conference of Education, Research and Innovation. Seville, 14th, 15th and 16th of November, 2016.

Giménez E., Garrido-Cárdenas J. A., Salmerón-Manzano E., y Manzano-Agugliaro F. (2016). Generic skills assessment in the environmental science degree at University of Almeria. ICERI 2016. 9th annual International Conference of Education, Research and Innovation. Seville, 14th, 15th and 16th of November, 2016.

González López, I. (2003). Determinación de los elementos que condicionan la calidad de la universidad: Aplicación práctica de un análisis factorial. Relieve (9)1, 83-96.

Jaramillo, A., Pineda, A. G., y Correa, J. S. O. (2012). Estudios sobre egresados la experiencia de la universidad EAFIT. Revista Universidad EAFIT, 42(141), 111-124.

León Benítez, M. R. (2007). La licenciatura de derecho en el contexto de la convergencia europea. Valencia: Tirant lo Blanch. 
MECD. 2015. Datos y Cifras del Sistema Universitario Español. Curso 2015-2016. Disponible en: http://www.mecd.gob.es/dms/mecd/educacion-mecd/areas-educacion/universidades/estadisticasinformes/datos-cifras/datos-y-cifras-SUE-2015-16-web.pdf

Manzano-Agugliaro, F., Salmerón-Manzano E., y Perea-Moreno A. J. (2016). Las competencias transversales de las universidades del Campus de Excelencia Agroalimentario (Cei-A3). Espiral. Cuadernos del Profesorado, 9(19), 68-77. Disponible en: http://espiral.cepcuevasolula.es/.

Rodríguez-Hidalgo, A. J., Villén, J. C., y Espejo, M. M. (2015). Desarrollo de Competencias en el Prácticum de Maestros: Abpy Coaching Multidimensional. Profesorado. Revista de Currículum y Formación de Profesorado, 19(1), 414-434.

Sobrinho, J. D. (2014). Universidad, conocimiento y construcción de un mundo nuevo. InterCambios: Dilemas y Transiciones de la Educación Superior, 1(1), 10-19.

Saldaña, M. N. (2011). Los Estudios de Género en los Grados en Derecho: Propuestas para un diseño curricular de la enseñanza del Derecho Constitucional con perspectiva de género en el Espacio Europeo de Educación Superior. Revista de Educación y Derecho (03). Disponible en http://revistes.ub.edu/index.php/RED/article/view/1781/1764.

Yániz, C. (2008). Las competencias en el currículo universitario: implicaciones para diseñar el aprendizaje y para la formación del profesorado. Red U. Revista de Docencia Universitaria, número monográfico $1^{\circ}$, consultado (28, 05, 2016) en http://www.redu.m.es/Red_U/m1.

Rey, B. (1996). Les compétences transversales en question. París. Ed. ESF.

Zabalza M. A. (2002) La enseñanza universitaria. El escenario y sus protagonistas. Madrid: Narcea.

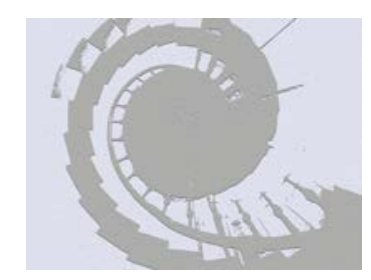

